\title{
Ovarian Teratoma or Uterine Malformation? PET/MRI as a Novel Useful Tool in NMDAR Encephalitis
}

\author{
FILIPPO CRIMİ $^{1}$, GIULIA CAMPORESE ${ }^{2}$, CARMELO LACOGNATA $^{1}$, \\ GIUSEPPE FANELLI ${ }^{3}$, DIEGO CECCHIN ${ }^{4}$ and MARCO ZOCCARATO ${ }^{2}$ \\ ${ }^{1}$ Radiology Unit, Department of Medicine - DIMED, University-Hospital Padua, Padua, Italy; \\ ${ }^{2}$ Neurology Unit, Ospedale Sant'Antonio, Padua, Italy; \\ ${ }^{3}$ Surgical Pathology \& Cytopathology Unit, Department of Medicine - \\ DIMED, University-Hospital of Padua, Padua, Italy; \\ ${ }^{4}$ Nuclear Medicine Unit, Department of Medicine DIMED, University-Hospital Padua, Padua, Italy
}

\begin{abstract}
This is a case report of a 17-year-old girl affected by N-methyl-D-aspartate-receptor (NMDAR) encephalitis suspected for a paraneoplastic syndrome. Ultrasound (US) and computed tomography (CT) imaging identified an ovarian lesion compatible with teratoma. ${ }^{18} \mathrm{~F}$ fluorodeoxyglucose (FDG) positron emission tomography/ magnetic resonance imaging (PET/MRI), performed to evaluate metabolic activity of the brain and of the ovarian mass, correctly changed the diagnosis to uterine malformation that was later histologically proven.
\end{abstract}

\section{Case Report}

A 17-year-old girl had been referred to our center for the development of focal seizures, confusion and behavioural disturbances. Laboratory testing showed no sign of viral or bacterial infections and brain magnetic resonance imaging (MRI) was normal. Serum and cerebrospinal fluid positivity for anti-N-Methyl-D-Aspartate receptor (NMDAR) antibodies led to the diagnosis of autoimmune encephalitis (1). To investigate the presence of malignancy, the patient underwent gynaecological ultrasound and abdominal contrast-enhanced CT that found a 2-cm cyst with thick wall and increased vascularization in the left ovary, arising the suspect of ovarian teratoma.

This article is freely accessible online.

Correspondence to: Filippo Crimì, Department of Medicine DIMED, Radiology section University of Padua, via Giustiniani 2, Padua, 35100, Italy. e-mail: filippo.crimi@uniroma1.com

Key Words: PET/MRI, NMDAR, encephalitis, ovarian teratoma, uterine malformation.
Therefore, a whole-body ${ }^{18}$ F-FDG PET-MRI scan was performed to explore brain metabolism and better characterize the ovarian mass. Slight MRI signal hyperintensity on FLAIR (Fluid Attenuated Inversion Recovery) sequence in the medial temporal lobes and a concomitant low uptake of ${ }^{18} \mathrm{~F}$-FDG in the amygdala bilaterally were detected. Whole-body PET/MRI showed a rounded solid lesion close to the left ovary with central hyper-intense core in T2 weighted axial and axialoblique images. The contrast-enhancement after gadolinium injection was similar to uterine body and glucose uptake of the lesion was lower than the metabolic activity of the ovaries (Figure 1).

Findings were therefore more suggestive of uterine malformation than of ovarian teratoma. Patient underwent pelvic laparoscopy that revealed a unicornuate right uterus with hypoplastic left uterine horn. The histological examination excluded any neoplasm and showed uterine tissue covered by endometrial epithelium with cystic ectasia and glandular sub-atrophy (Figure 2).

Anti-NMDAR encephalitis is an autoimmune disorder that may occur in young women and is related to autoantibodies against NR1 subunit of NMDA receptor. Clinical manifestation consists of confusion and memory deficits, psychosis, seizures, movement disorders and impaired consciousness.

In a certain number of cases, especially in young women, anti-NMDAR encephalitis is associated to neoplasms, mostly ovarian teratoma $(2,3)$. In these cases, antibody production is probably due to reaction against ectopic neural antigens expressed by the tumor.

Among uterine abnormalities, rudimental hypo-plastic uterine horns (4) can be misinterpreted as ovarian masses especially if not associated with hematometra.

Ultrasound diagnosis of teratoma is not always easy because of absence of typical features in smaller lesions and it requires the use of other imaging techniques (5). 

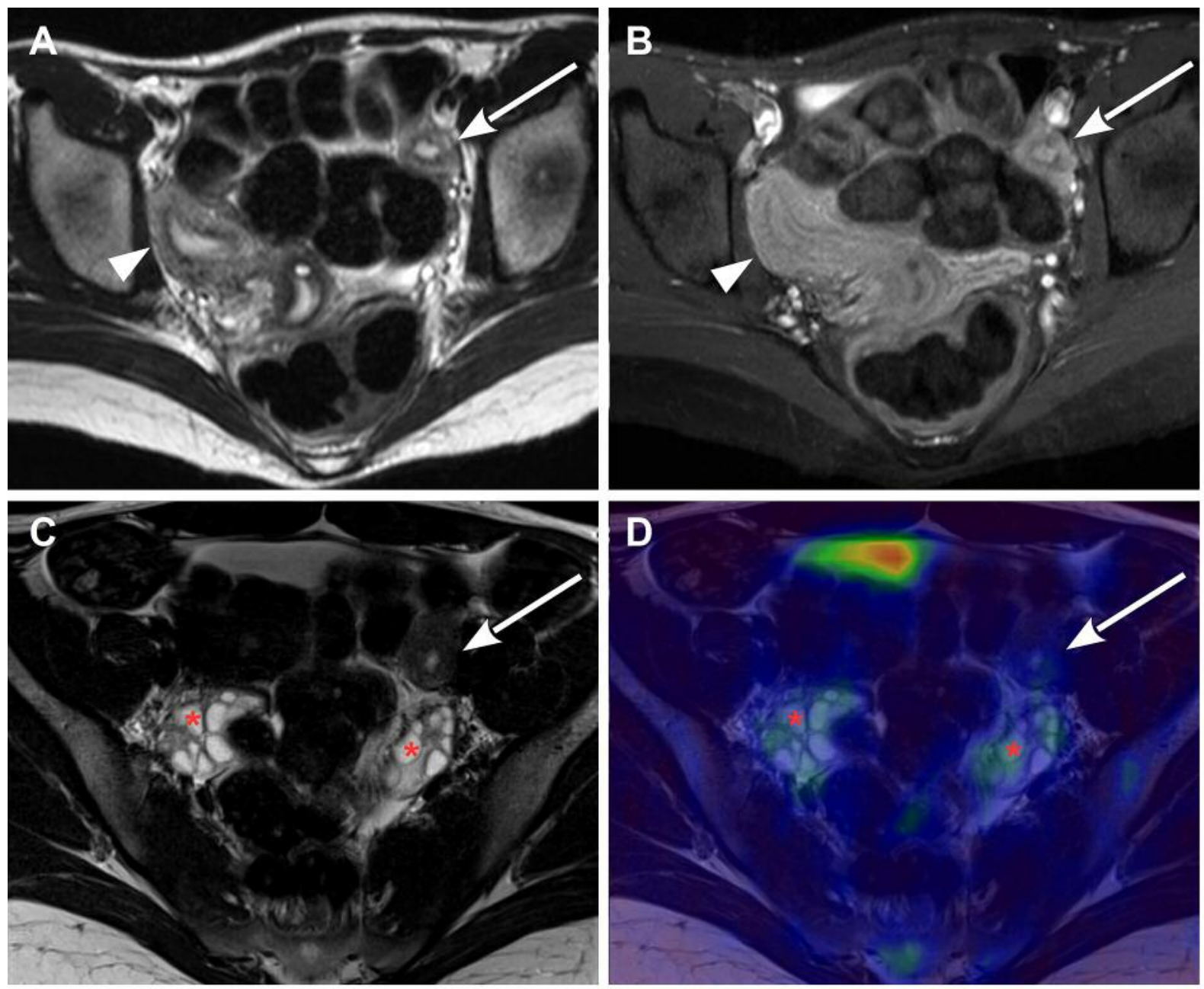

Figure 1. ${ }^{18}$ F-FDG PET/MRI images of the pelvis. A) Axial MRI scan (T2 weighted) showing rounded solid formation (white arrow) close to the left ovary with central hyper-intense core and right uterine horn (white arrowhead); B) Axial MRI scan (T1 weighted with fat saturation after gadolinium injection) showing moderate contrast enhancement of the solid lesion (white arrow) and right uterine horn with similar enhancement pattern (white arrowhead); C) Para-axial MRI scan (T2 weighted) showing the mass (white arrow) and the two ovaries (red asterisks); D) Fused PET/MRI showing no significant metabolic activity of the lesion (white arrow) and mild physiologic metabolism of the ovaries (red asterisks).

CT and MRI are pivotal in radiological diagnosis: CT can identify calcification within the mass but MRI resulted to be more accurate for ovarian teratoma diagnosis and for müllerian duct anomalies assessment (5-7). Whole-body PET/CT demonstrated high accuracy in staging antiNMDAR encephalitis and in detecting correlated ovarian malignancies $(8,9)$.

The combination of PET and MRI in the same procedure merges the high anatomical details of MRI with the fundamental metabolic information of FDG-PET, but probably due to the low availability of this technique no case of autoimmune encephalitis has been reported so far.

This patient was affected with a non-paraneoplastic form of encephalitis and completely recovered after administration of

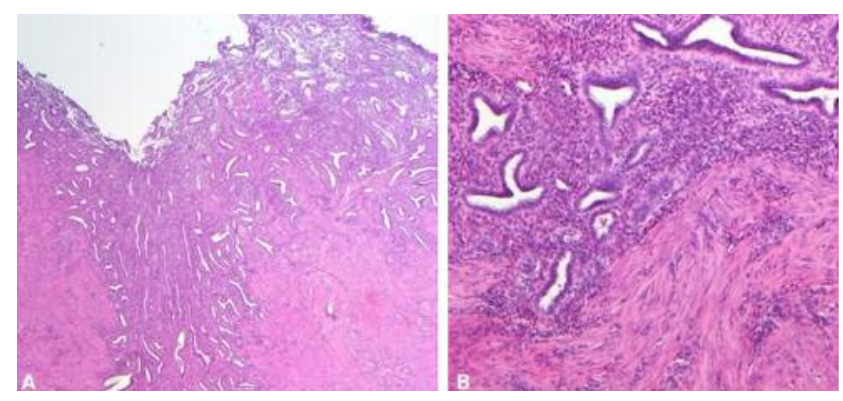

Figure 2. Histopathological examination. Hematoxylin and eosin staining of the surgically removed left solid formation $(25 x$ and $100 x$ magnification in $A$ and $B$ respectively) showing uterine myometrium covered by endometrial epithelium with cystic ectasia and glandular sub-atrophy. 
plasma-exchange and rituximab. Exclusion of paraneoplastic origin was challenging because of the particular shape and position of the hypo-plastic left uterine horn.

In conclusion, combined ${ }^{18} \mathrm{~F}-\mathrm{FDG}$ PET/MRI can be a useful "all-in-one" tool in evaluating both anti-NMDA receptor encephalitis brain changes and to screen with good accuracy possible associated malignancies (1-10).

\section{Conflicts of Interest}

The Authors have indicated they have no potential conflicts of interest to disclose.

\section{References}

1 Kayser MS and Dalmau J: Anti-NMDA receptor encephalitis, autoimmunity, and psychosis. Schizophr Res 176(1): 36-40, 2016.

2 Florence NR and Dalmau J: Anti-N-Methyl-D-Aspartate Receptor (NMDAR) Encephalitis in children and adolescents. Ann Neurol 66: 11-18, 2009.

3 Di Spiezio Sardo A, Campo R, Gordts S, Spinelli M, Cosimato C, Tanos V, Brucker S, Li TC, Gergolet M, De Angelis C, Gianaroli L and Grimbizis G: The comprehensiveness of the ESHRE/ESGE classification of female genital tract congenital anomalies: a systematic review of cases not classified by the AFS system. Hum Reprod 30(5): 1046-1058, 2015.

4 Outwater EK, Siegelman ES and Hunt JL: Ovarian teratomas: tumor types and imaging characteristics. Radiographics 21(2): 475-490, 2001.
5 Epelman M, Chikwava KR, Chauvin N and Servaes S: Imaging of pediatric ovarian neoplasms. Pediatr Radiol 41(9): 1085-1099, 2011.

6 Li Y, Phelps A, Zapala MA, MacKenzie JD, MacKenzie TC and Courtier J: Magnetic resonance imaging of Müllerian duct anomalies in children. Pediatr Radiol 46(6): 796-805, 2016.

7 Nakamoto R, Nakamoto Y, Ishimori T, Fushimi Y and Togashi $\mathrm{K}$ : ${ }^{18} \mathrm{~F}$-fluorodeoxyglucose uptake in anti-n-methyl-d-aspartatereceptor encephalitis associated with an immature teratoma. Clin Nucl Med 42(2): 157-160, 2017.

8 Basu S and Rubello D: PET imaging in the management of tumors of testis and ovary: current thinking and future directions. Minerva Endocrinol 33(3): 229-256, 2008.

9 Graus F, Titulaer MJ, Balu R, Benseler S, Bien CG, Cellucci T, Cortese I, Dale RC, Gelfand JM, Geschwind M, Glaser CA, Honnorat J, Höftberger R, Iizuka T, Irani SR, Lancaster E, Leypoldt F, Prüss H, Rae-Grant A, Reindl M, Rosenfeld MR, Rostásy K, Saiz A, Venkatesan A, Vincent A, Wandinger KP, Waters $\mathrm{P}$ and Dalmau J:A clinical approach to diagnosis of autoimmune encephalitis. Lancet Neurol 15: 391-404, 2016.

10 Day BK, Eisenman L, Black J, Maccotta L and Hogan RE: A case study of voltage-gated potassium channel antibody-related limbic encephalitis with PET/MRI findings. Epilepsy Behav Case Rep 4: 23-26, 2015. 